\title{
Co-treatment With HIV Protease Inhibitor Nelfinavir Greatly Increases Late-phase Apoptosis of Drug-resistant KBV20C Cancer Cells Independently of P-Glycoprotein Inhibition
}

\author{
JI YEONG KIM*, YOO JUNG PARK*, BYUNG-MU LEE and SUNGPIL YOON \\ School of Pharmacy, Sungkyunkwan University, Suwon, Republic of Korea
}

\begin{abstract}
Background/Aim: The study focused on identifying the mechanisms or drugs that might sensitize resistant KBV20C human oral squamous carcinoma cells overexpressing $P$-glycoprotein $(P-g p)$ to antimitotic drug treatment. Materials and Methods: Five HIV protease inhibitors (atazanavir, nelfinavir, darunavir, lopinavir, and ritonavir) were tested to identify drugs that could be used at a relatively low dose for sensitizing antimitotic drug-resistant KBV2OC cells. Fluorescence-activated cell sorting, annexin $V$ analyses, and rhodamine uptake tests were performed to further investigate the mechanism of action. Results: Co-treatment with nelfinavir or lopinavir had a high sensitizing effect on vincristine-treated KBV20C cells. Nelfinavir and lopinavir reduced cell viability, increased $G_{2}$ phase arrest, and up-regulated apoptosis when used as a co-treatment with vincristine. We also demonstrated that eribulin co-treatment with nelfinavir and lopinavir similarly increased sensitization of KBV20C cells. Only lopinavir was found to have a high P-gp-inhibitory activity (similar to verapamil). Interestingly, nelfinavir had very low P-gp-inhibitory activity, suggesting that vincristine-nelfinavir sensitization is independent of the P-gp-inhibitory effect of nelfinavir. We also demonstrated this same combination mainly caused sensitization due to late apoptosis in P-gpoverexpressing drug-resistant KBV20C cells. Conclusion: Highly antimitotic drug-resistant KBV20C cells can be sensitized by co-treatment with the repositioned HIV protease inhibitors nelfinavir and lopinavir. In particular, the sensitizing effect of co-treatment with nelfinavir on antimitotic drugresistant cancer cells was found to be strong and independent
\end{abstract}

*These Authors contributed equally to this study.

Correspondence to: Sungpil Yoon, Ph.D., School of Pharmacy, Sungkyunkwan University, 2066 Seobu-ro, Jangan-gu, Suwon, Gyeonggi-do, 16419, Republic of Korea. Tel: +82 1055024893, Fax: +82 312928800, e-mail: syoon88@gmail.com

Key Words: Nelfinavir, lopinavir, HIV protease inhibitors, repositioning drug, cancer, $\mathrm{P}$-gp, drug resistance. of P-gp-inhibitory activity. As P-gp inhibition can be toxic to normal cells, selecting nelfinavir may be safer for normal cells in patients with drug-resistant cancer.

Antimitotic drugs inhibit mitosis by targeting microtubules and preventing their polymerization or depolymerization. Paclitaxel, docetaxel, vincristine, vinorelbine, vinblastine and eribulin are examples of antimitotic drugs (1-4). Although antimitotic drugs are widely used in cancer treatment, cancer cells can develop resistance to these drugs in various ways.

Overexpression of $\mathrm{P}$-glycoprotein (P-gp) is one wellknown mechanism of antimitotic drug resistance. $\mathrm{P}$-gp is a membrane channel that can pump out antimitotic drugs and thus avoid cell drug-induced toxicity (5-8). Identifying sensitizing mechanisms or effective drugs for cancer cells that overexpress P-gp would lead to better treatment of patients who develop resistance to antimitotic drugs. Although P-gp inhibitors have been developed, toxicity in normal cells resulted in the failure of clinical testing. Therefore, it is also important to investigate novel therapies without P-gp inhibition for P-gp-overexpressing drugresistant cancer cells.

In this study, we aimed to identify novel drugs that might be repositioned for their possible application to $\mathrm{P}$-gpoverexpressing resistant cancer cells, including increased sensitization efficacy of such drugs when used in combination with antimitotic drugs. The urgent need for pharmacological treatments for P-gp-overexpressing drug-resistant cancer can be efficiently addressed if novel mechanisms of using repositioned drugs are identified because these drugs can be used without the need for further toxicity evaluation. Repositioned drugs that can sensitize P-gp-overexpressing drug-resistant cancer cells have been reported (9-11).

In our preliminary studies (unpublished data), we found that an HIV protease inhibitor had potential sensitization effects when co-administered with an antimitotic drug. It has been reported that the effectiveness of HIV protease inhibitors correlates with P-gp expression or activity (12-17). For example, HIV protease inhibitors that are substrates of P-gp can induce resistance in patients with $\operatorname{HIV}(16,18)$. HIV protease 
inhibitors can increase resistance by inducing overexpression of $\mathrm{P}$-gp in cells, which reduces both toxicity and efficacy of the drug. In contrast, studies showed that an HIV protease inhibitor can play a role as a P-gp blocker $(12,14)$. An HIV protease inhibitor also showed P-gp-inhibitory activity in resistant cancer cells and sensitized them to co-treatment with doxorubicin (19). However, a comparison of HIV protease inhibitors and their exact mechanisms has not been reported. In this study, based on a literature search for HIV protease inhibitors, we found five candidates: Atazanavir, nelfinavir, darunavir, lopinavir, and ritonavir. We then investigated which HIV protease inhibitors have a relatively low half-maximal inhibitory concentration $\left(\mathrm{IC}_{50}\right)$ for sensitizing P-gp-overexpressing drug-resistant KBV20C cancer cells.

As these drugs are already in use in humans, we hope our findings will contribute to the development of HIV protease inhibitor-based therapies for the co-treatment of highly drugresistant tumors.

\section{Materials and Methods}

Reagents and cell culture. Rhodamine123 (rhodamine), fluphenazine, and verapamil were purchased from Sigma-Aldrich (St. Louis, MO, USA). Vincristine was purchased from Enzo Life Sciences (Farmingdale, NY, USA). Atazanavir, nelfinavir, darunavir, lopinavir, and ritonavir were purchased from Selleckchem (Houston, TX, USA). Aqueous solutions of eribulin (halaven; Eisai Korea, Seoul, South Korea) were obtained from the National Cancer Center in South Korea.

Human oral squamous carcinoma cell line KB and its multidrugresistant subline KBV20C were obtained from Dr. Yong Kee Kim (College of Pharmacy, Sookmyung Women's University, Seoul, South Korea) and have been previously described (20-23). All cell lines were cultured in RPMI 1640 containing $10 \%$ fetal bovine serum, $100 \mathrm{U} / \mathrm{ml}$ penicillin, and $100 \mu \mathrm{g} / \mathrm{ml}$ streptomycin (WelGENE, Daegu, South Korea).

Microscopic observation. Cells were grown to $40-50 \%$ confluence in $60-\mathrm{mm}$ diameter dishes and treated with $5 \mu \mathrm{M}$ atazanavir, nelfinavir, darunavir, lopinavir, ritonavir or fluphenazine, $10 \mu \mathrm{M}$ verapamil, $50 \mathrm{ng} / \mathrm{ml}$ eribulin, alone or combined with $5 \mathrm{nM}$ vincristine for $24 \mathrm{~h}$. The medium was removed, and phosphatebuffered saline (PBS) was added to each dish. Attached cells were examined immediately in two independent experiments using an ECLIPSE Ts2 inverted microscope (Nikon, Tokyo, Japan) with a $4 \times$ or a $10 \times$ objective lens (Nikon Microscopy U).

Rhodamine uptake tests. The tests used to assess the ability of a drug to inhibit P-gp were based on a previously described method (20-23). Briefly, cells were grown to $40-50 \%$ confluence in $60-\mathrm{mm}$ diameter dishes and treated with $5 \mu \mathrm{M}$ atazanavir, nelfinavir, lopinavir or ritonavir, alone or combined with $10 \mu \mathrm{M}$ verapamil and incubated for $24 \mathrm{~h}$ or $4 \mathrm{~h}$ at $37^{\circ} \mathrm{C}$. Cells were then incubated with $2 \mu \mathrm{g} / \mathrm{ml}$ rhodamine for $1 \mathrm{~h} 30 \mathrm{~min}$ at $37^{\circ} \mathrm{C}$. The medium was removed, and the cells were washed with PBS. The stained cells were then analyzed in two independent experiments using a Guava EasyCyte Plus Flow Cytometer (Merck Millipore, Burlington, MA, USA).
Fluorescence-activated cell sorting (FACS) analysis. FACS analysis was performed as previously described (23-26). Cells were grown to $40-50 \%$ confluence in $60-\mathrm{mm}$ diameter dishes and treated with 5 $\mu \mathrm{M}$ atazanavir, nelfinavir, lopinavir or ritonavir, alone or combined with $5 \mathrm{nM}$ vincristine for $24 \mathrm{~h}$. The cells were then dislodged by trypsin and pelleted by centrifugation. The pelleted cells were washed thoroughly with PBS, suspended in $75 \%$ ethanol for at least $1 \mathrm{~h}$ at $4^{\circ} \mathrm{C}$, washed with PBS, and re-suspended in a cold propidium iodide (PI) staining solution $(100 \mu \mathrm{g} / \mathrm{ml} \mathrm{RNase}$ A and $50 \mu \mathrm{g} / \mathrm{ml} \mathrm{PI}$ in PBS) for $30 \mathrm{~min}$ at $37^{\circ} \mathrm{C}$. The stained cells were analyzed in two independent experiments for relative DNA content using a Guava EasyCyte Plus Flow Cytometer (Merck Millipore, Burlington, MA, USA).

Annexin $V$ analysis. Annexin $\mathrm{V}$ analysis was conducted by using annexin V-fluorescein isothiocyanate (FITC) staining kit (BD Bioscience, Franklin, NJ, USA) as previously described (23-26). Cells were grown to $40 \%-50 \%$ confluence in $60-\mathrm{mm}$ diameter dishes and treated with $5 \mu \mathrm{M}$ atazanavir, nelfinavir, lopinavir or ritonavir, or $10 \mu \mathrm{M}$ verapamil, alone or combined with $5 \mathrm{nM}$ vincristine for $24 \mathrm{~h}$. The cells were then dislodged by trypsin and pelleted by centrifugation. The pelleted cells were washed with PBS. Cells in $100 \mu \mathrm{l}$ of binding buffer received $5 \mu \mathrm{l}$ of Annexin VFITC and $5 \mu \mathrm{l}$ of PI and were, then, incubated for $15 \mathrm{~min}$ at room temperature. The stained cells were analyzed in two independent experiments using a Guava EasyCyte Plus Flow Cytometer (Merck Millipore, Burlington, MA, USA).

Cell viability assay. Cell proliferation was measured by a colorimetric assay using the EZ-CyTox cell viability assay kit (Daeillab, South Korea) according to the manufacturer's instructions. Briefly, cells were grown to $30-40 \%$ confluence in wells of 96-well plates and treated with $5 \mu \mathrm{M}$ atazanavir, nelfinavir, darunavir, lopinavir or ritonavir, or $10 \mu \mathrm{M}$ verapamil, alone or combined with $5 \mathrm{nM}$ vincristine for $48 \mathrm{~h}$. They were then incubated with $10 \mu \mathrm{l}$ of EZ-CyTox solution for $1-2 \mathrm{~h}$ at $37^{\circ} \mathrm{C}$. Absorbance at $450 \mathrm{~nm}$ was determined immediately using a VERSA MAX Microplate Reader (Molecular Devices Corp., Sunnyvale, CA, USA). All experiments were performed at least in triplicate and repeated twice.

Statistical analysis. Data are presented as the mean \pm standard deviation (S.D.). Statistical analysis was performed by using Student's $t$-test and one-way analysis of variance (ANOVA) followed by a multiple-comparison test. Results were considered statistically significant compared to those of the control when $p<0.05$.

\section{Results}

Nelfinavir and lopinavir sensitize vincristine-treated resistant KBV20C cancer cells better than other HIV protease inhibitors. We aimed to identify drugs that could be repositioned to sensitize resistant cells, or improve efficacy when used in combination with chemotherapeutic drugs. In our preliminary screening, we found that an HIV protease inhibitor increased sensitization to vincristine treatment of resistant cancer cells. In this study, we focused on which HIV protease inhibitors combine well with antimitotic drugs for Pgp-overexpressing resistant cancer cells, then we investigated the mechanisms of sensitization. We used vincristine, an 

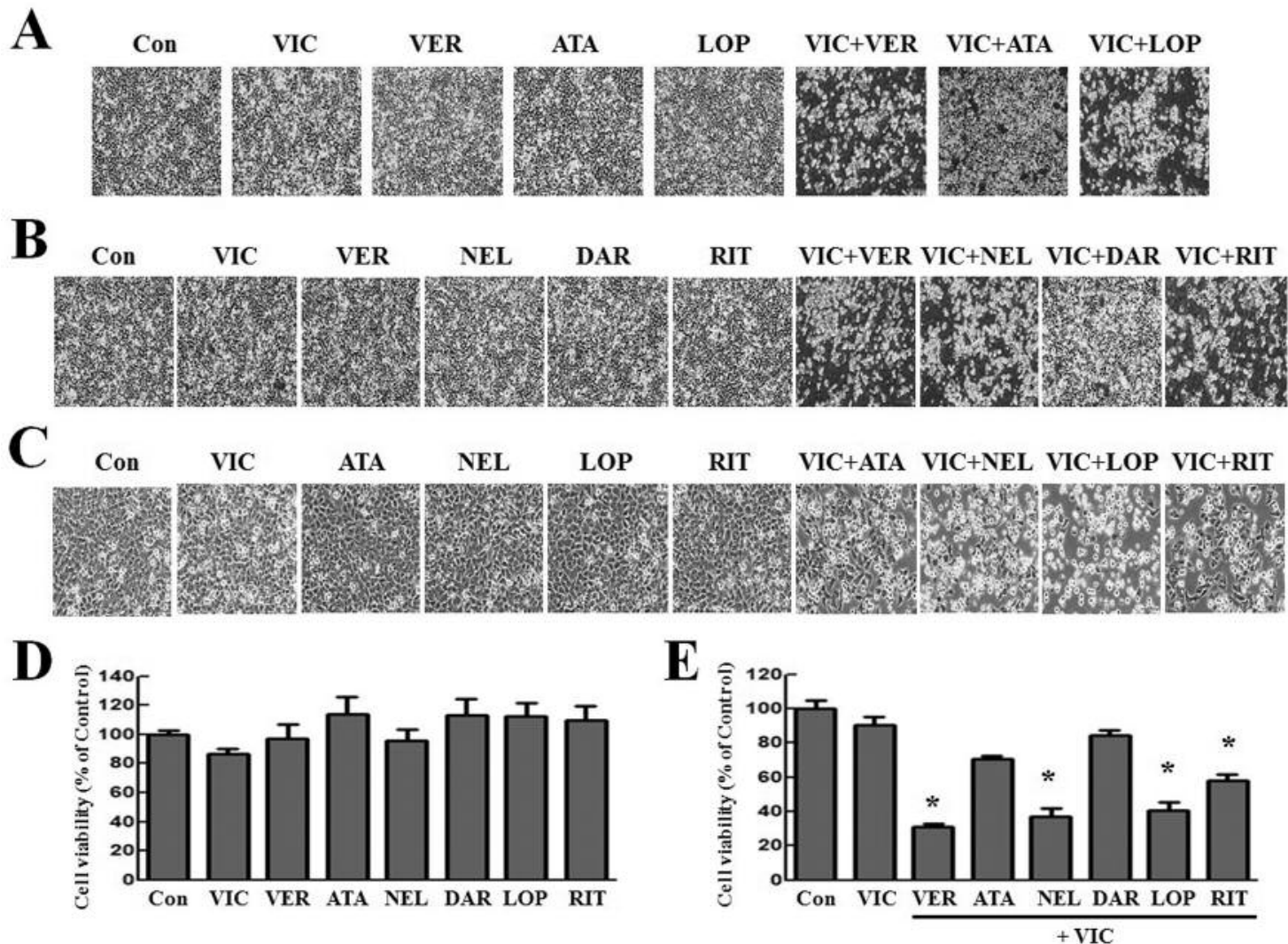

Figure 1. Nelfinavir and lopinavir better sensitized vincristine-resistant KBV20C cancer cells than did other HIV protease inhibitors. A-C: KBV20C cells were grown on $60 \mathrm{~mm}$-diameter dishes and treated with $5 \mathrm{nM}$ vincristine (VIC), $10 \mu \mathrm{M}$ verapamil (VER), $5 \mu M$ atazanavir (ATA), $5 \mu M$ nelfinavir (NEL), $5 \mu M$ darunavir (DAR), $5 \mu M$ lopinavir (LOP), or $5 \mu M$ ritonavir (RIT) alone and in combination with $5 \mathrm{nM}$ vincristine, or with $0.1 \%$ dimethyl sulfoxide (Con). After 1 day, cells were examined using an inverted microscope $\times 4$ magnification (A and B) or $\times 10$ magnification (C). D and E: KBV20C cells were plated on 96-well plates and grown to 30\%-40\% confluence. The cells were then treated as described above for $48 \mathrm{~h}$. Cell viability assay was then performed as described in the Materials and Methods. The data are presented as the mean \pm S.D. of at least two experiments repeated in triplicate experiments. Statistical analysis was conducted using one-way analysis of variance (ANOVA) followed by multiplecomparison test. $*$ Significantly different at $p<0.05$ compared to the corresponding control.

antimitotic drug that is routinely used as a chemotherapeutic agent in patients with cancer $(27,28)$ KBV20C cancer cells express a vincristine-resistant phenotype via P-gp overexpression $(21,22)$. We compared the sensitizing effects of verapamil as a positive control, which is a P-gp inhibitor (5), and it is well-known that co-treatment with verapamil increases $\operatorname{KBV} 20 \mathrm{C}$ cell sensitivity to vincristine $(21,28)$.

As shown in Figure 1A-C with microscopic observations, $5 \mu \mathrm{M}$ of nelfinavir, lopinavir, or ritonavir sensitized cells to vincristine, whereas $5 \mu \mathrm{M}$ of atazanavir or darunavir had slight or no sensitizing effect. When we compared the effects to $10 \mu \mathrm{M}$ verapamil as a positive control, similar sensitization was produced in cells co-treated with vincristine, reducing KBV20C cell numbers and suggesting that a lower dose of nelfinavir, lopinavir, or ritonavir is sufficient and as effective as verapamil in sensitizing $\mathrm{P}$-gp-overexpressing resistant cancer cells. We performed a more detailed quantitative analysis with a cell viability test. Whereas there was no decrease in viability with single-drug treatment (Figure 1D), we found that nelfinavir and lopinavir highly reduced viability of vincristine-treated KBV20C cells, almost as much as did the combination with verapamil (Figure 1E). The results demonstrated that both nelfinavir and lopinavir are as effective as verapamil in sensitizing vincristine-resistant cancer cells. In the viability test, ritonavir had a slightly weaker sensitizing effect compared with that of nelfinavir and lopinavir (Figure 1E). We also confirmed the results by apoptotic analysis using annexin $\mathrm{V}$ staining (Figure 2A). Apoptotic cells in both early and late phases made up almost $30 \%$ of cells on vincristine-nelfinavir or vincristine-lopinavir co-treatment, whereas with vincristine-ritonavir it was less than $20 \%$ (Figure 2A). This also suggests that both nelfinavir and lopinavir can be used at a low dose, with reduced drug toxicity, to sensitize vincristine-resistant cancer cells. We found both nelfinavir and lopinavir to have similar or better efficacy compared to that of verapamil, and compared to 
A

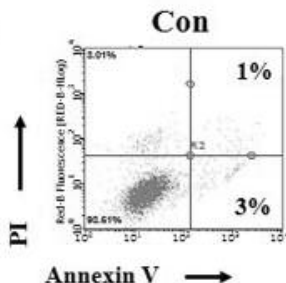

Annexin V $\longrightarrow$

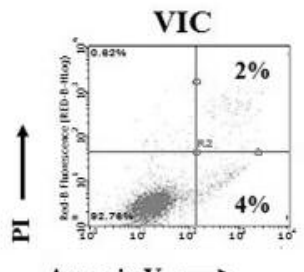

Annexin V $\longrightarrow$
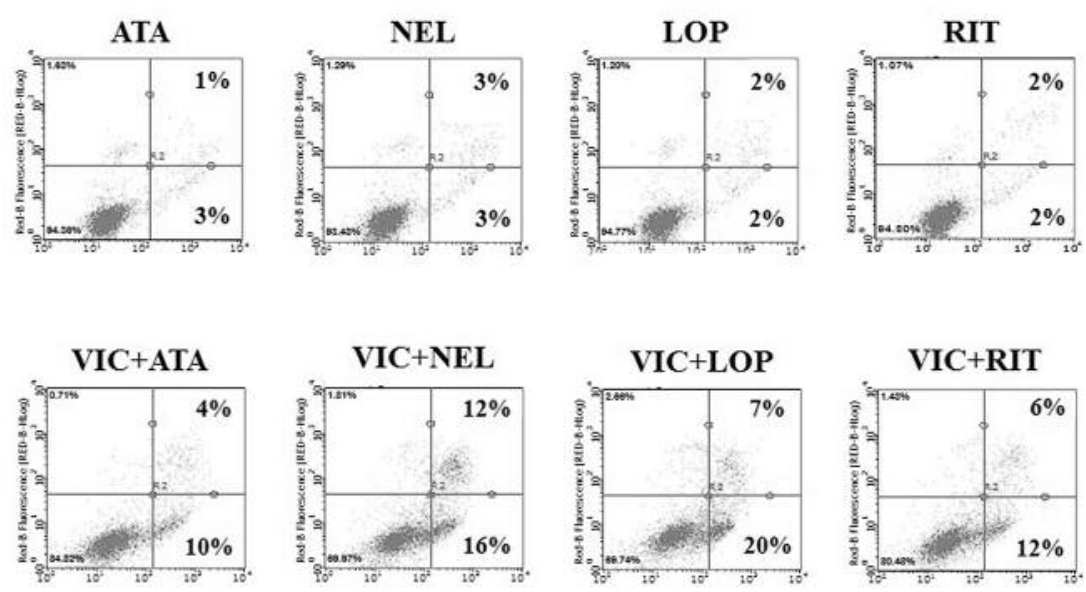

B
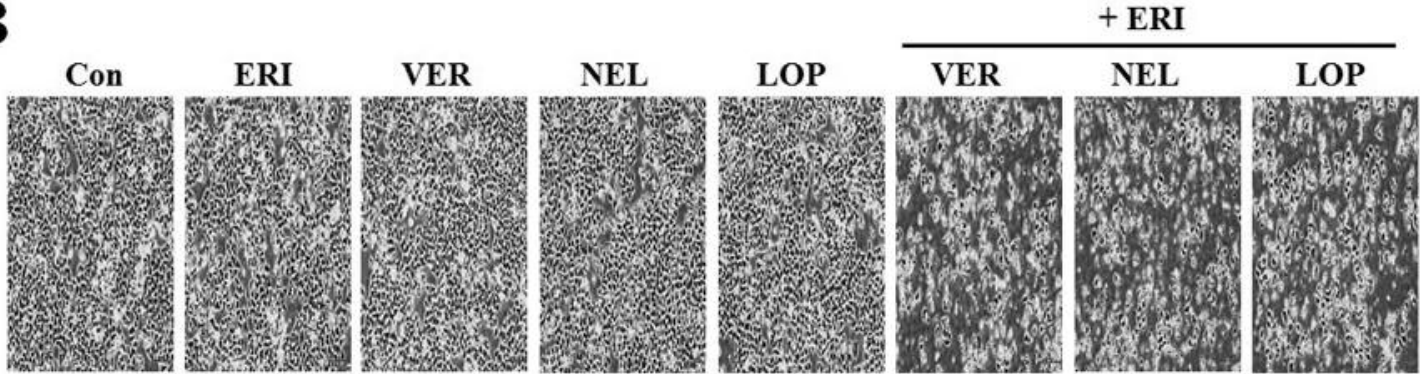

Figure 2. Both nelfinavir (NEL) and lopinavir (LOP) at low doses also increased sensitization of KBV20C cells to antimitotic drug. A: KBV20C cells were grown on $60 \mathrm{~mm}$-diameter dishes and stimulated with $5 \mu \mathrm{M}$ of atazanavir (ATA), nelfinavir, lopinavir, or ritonavir (RIT) alone and in combination with $5 \mathrm{nM}$ vincristine, or with $0.1 \%$ dimethyl sulfoxide (Con). After 24 h, annexin V analyses were performed as described in Materials and Methods. B: KBV20C cells were grown on $60 \mathrm{~mm}$-diameter dishes and treated with $50 \mathrm{ng} / \mathrm{ml}$ eribulin (ERI), $10 \mu \mathrm{M}$ verapamil, $5 \mu \mathrm{M}$ nelfinavir or $5 \mu \mathrm{M}$ lopinavir alone and in combination with $50 \mathrm{ng} / \mathrm{ml}$ eribulin, or with $0.1 \%$ dimethyl sulfoxide (Con). After 1 day, cells were examined using an inverted microscope at $\times 4$ magnification.

other HIV protease inhibitors, to have a higher sensitizing effect, with increased apoptosis.

Both nelfinavir and lopinavir at low doses increase sensitization of KBV20C cells treated with other anti-mitotic drugs. We also investigated whether nelfinavir and lopinavir were effective in combination with other antimitotic drugs. We tested eribulin, another antimitotic drug that has been recently developed and used in the treatment of metastatic cancer (29, $30)$. In previous work, we found that the KBV20C cell line is a very useful model of highly eribulin-resistant cancer (20, 26). As seen in Figure 2B, $5 \mu \mathrm{M}$ of nelfinavir or lopinavir produced similar sensitizing effects when combined with eribulin compared with vincristine-nelfinavir or vincristinelopinavir co-treatments. The eribulin-verapamil co-treatment at the same dose also had similar effects to vincristineverapamil co-treatment (Figure 2B). These results demonstrate that nelfinavir and lopinavir are also effective in sensitizing resistant cancer cells to co-treatment with eribulin. This finding also suggests that nelfinavir or lopinavir at low dose can be combined other antimitotic drugs to sensitize P-gpoverexpressing cancer cells.

Nelfinavir and lopinavir strongly induce G2 arrest of vincristinetreated resistant KBV20C cells. In order to further clarify the mechanism of action of vincristine-HIV protease inhibitor cotreatments, we performed FACS analyses. As shown in Figure $3 \mathrm{~A}$, all co-treatments of vincristine with nelfinavir, lopinavir, and ritonavir greatly increased the number of cells in $\mathrm{G}_{2}$ arrest compared with that observed after monotherapy with either agent. When quantifying cells in $\mathrm{G}_{2}$ arrest, we found that vincristine-nelfinavir and vincristine-lopinavir co-treatments were more effective than that of vincristine-ritonavir (Figure 3A). Vincristine-atazanavir co-treatment also had a slight effect on $\mathrm{G}_{2}$ arrest, suggesting that atazanavir has a slightly synergistic effect on vincristine-treated KBV20C cells. This indicates that an increase in cell-cycle arrest stimulated apoptosis, as apoptosis with vincristine-nelfinavir and vincristine-lopinavir co-treatment 

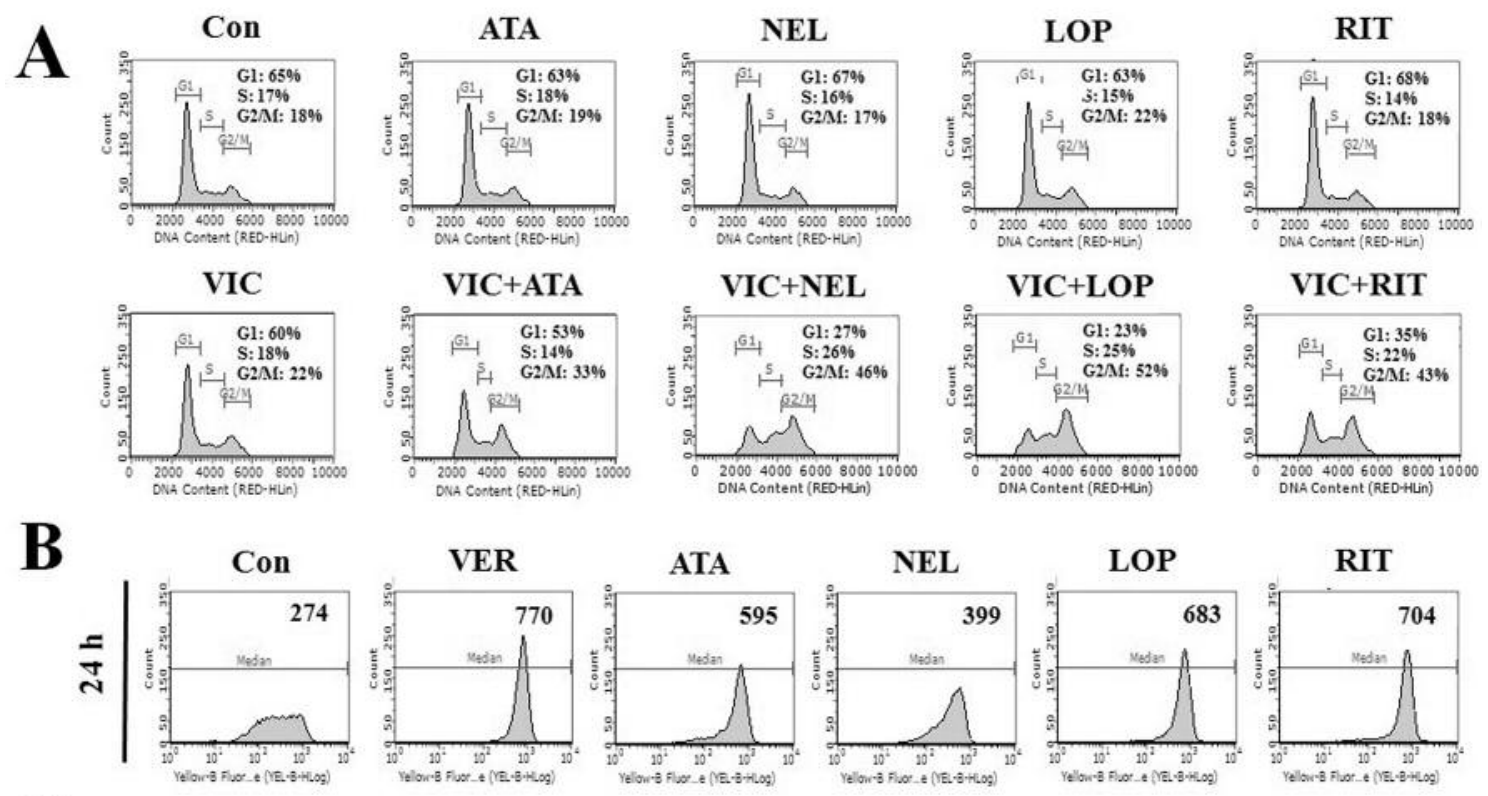

C
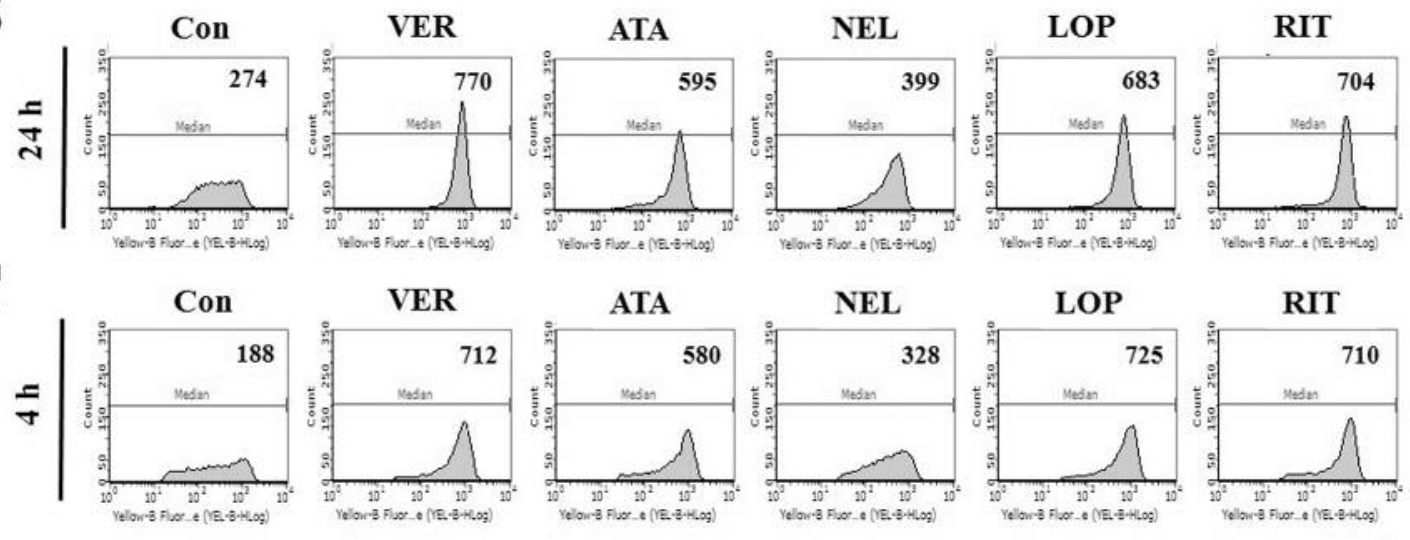

Figure 3. Nelfinavir (NEL) had low P-glycoprotein (P-gp)-inhibitory activity, whereas lopinavir (LOP) had high P-gp-inhibitory activity in KBV20C cells. A: KBV2OC cells were grown on $60 \mathrm{~mm}$-diameter dishes and treated with $5 \mu$ M atazanavir (ATA), nelfinavir, lopinavir or ritonavir (RIT) alone and in combination with $5 \mathrm{nM}$ vincristine, or with $0.1 \%$ dimethyl sulfoxide (Con). After $24 \mathrm{~h}$, fluorescence-activated cell sorting (FACS) analyses were performed to determine the cell-cycle distribution as described in the Materials and Methods. B and C: KBV20C cells were grown on $60 \mathrm{~mm}$-diameter dishes and treated with single agents as above or $10 \mu \mathrm{M}$ verapamil (VER). After 4 or $24 \mathrm{~h}$, live cells were stained with rhodamine and examined using FACS analysis, as described in the Materials and Methods.

was higher than with vincristine-ritonavir. Overall, HIV protease inhibitors increased apoptosis of vincristine-treated resistant KBV20C cells via $\mathrm{G}_{2}$ cell-cycle arrest.

Nelfinavir has low P-gp-inhibitory activity, whereas lopinavir has high P-gp-inhibitory activity. Next, we tested the P-gp-inhibitory activity of HIV protease inhibitors in Pgp-overexpressing KBV20C cells because we assumed that the differences in the degree of P-gp inhibition among HIV protease inhibitors were responsible for the difference in their sensitizing efficacy of vincristine-treated KBV20C cells. However, as shown in Figure 3B, P-gp inhibition by nelfinavir was much lower than that of verapamil, a wellknown P-gp inhibitor, and only slightly higher than that of the control (Figure 3B). In addition, nelfinavir showed lesser P-gp-inhibitory activity than atazanavir and ritonavir. Vincristine-nelfinavir sensitization was much higher than that of vincristine-atazanavir or vincristine-ritonavir (Figure 1), which indicates that sensitization by vincristine-nelfinavir of
KBV20C cells is minimally affected by the P-gp-inhibitory effects of nelfinavir. When we analyzed P-gp-inhibitory activity of lopinavir and ritonavir, we found that they have similar P-gp-inhibitory activity to verapamil, suggesting that P-gp inhibition by lopinavir and by ritonavir plays a major role in sensitizing in vincristine-lopinavir and vincristineritonavir co-treatment. As shown in Figure 3C, treatment with HIV protease inhibitors or verapamil for $4 \mathrm{~h}$ led similar results to those obtained with $24 \mathrm{~h}$ of treatment. This suggests that lopinavir and ritonavir inhibited P-gp via direct binding, similarly to the inhibitory mechanism of verapamil. Although both lopinavir and ritonavir had similarly high Pgp-inhibitory activity (Figure $3 \mathrm{~B}$ and $\mathrm{C}$ ), we found that the vincristine-lopinavir combination had a much better sensitizing effect on resistant cancer cells than vincristineritonavir co-treatment (Figures $1 \mathrm{E}$ and $2 \mathrm{~A}$ ). This suggests that lopinavir has both cytotoxic and P-gp-inhibitory effects on vincristine-treated KBV20C cells, whereas ritonavir only has P-gp-inhibitory activity. It seems that lopinavir and 

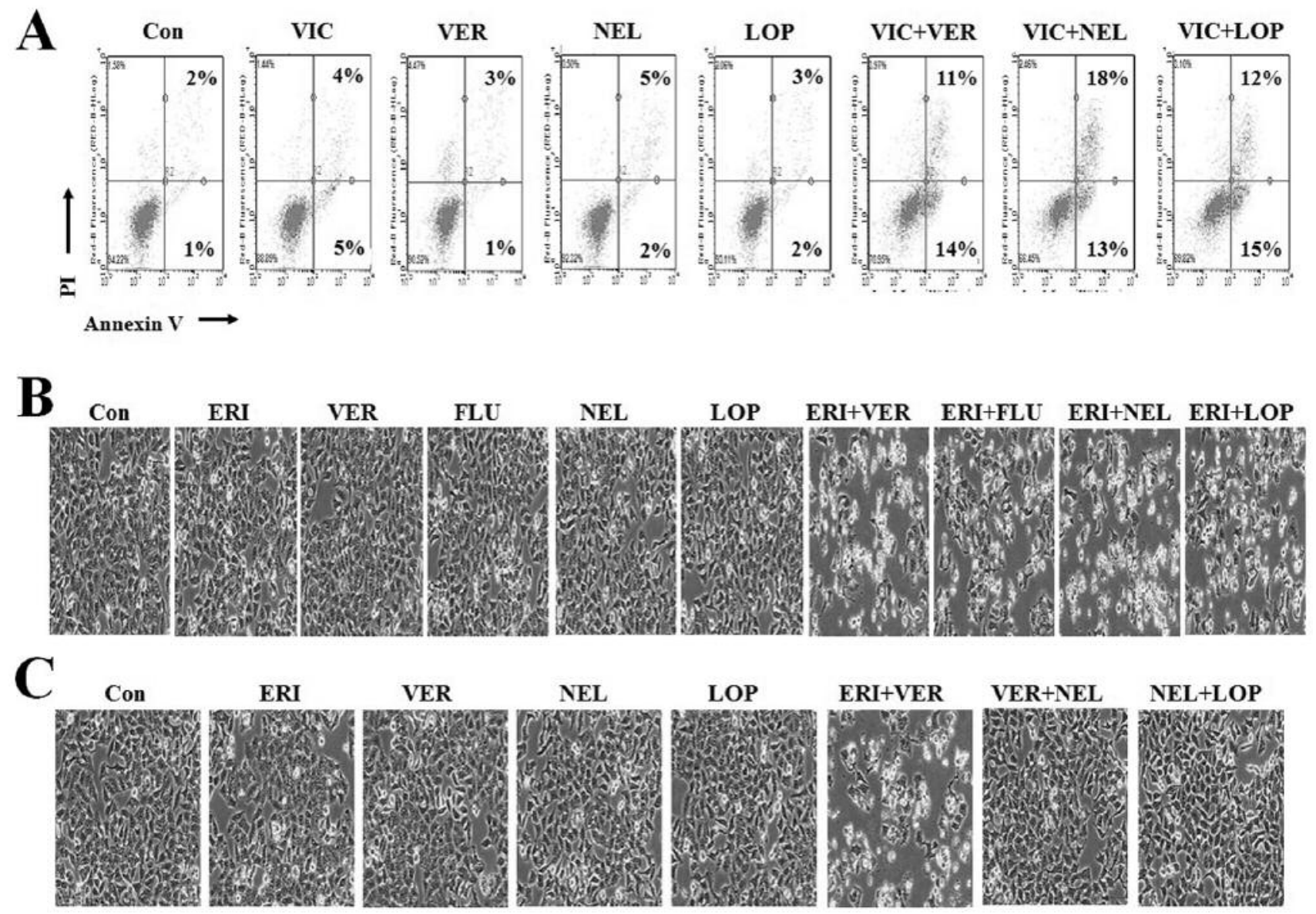

Figure 4. Co-treatment of resistant KBV20C cells with vincristine (VIC) and nelfinavir (NEL) increased late apoptosis, whereas co-treatment with lopinavir (LOP) increased early apoptosis. A: KBV20C cells were grown on $60 \mathrm{~mm}$-diameter dishes and treated with $10 \mu M$ verapamil (VER), $5 \mu \mathrm{M}$ nelfinavir, or $5 \mu \mathrm{M}$ lopinavir alone and in combination with $5 \mathrm{nM}$ vincristine, or with $0.1 \%$ dimethyl sulfoxide (Con). After $24 \mathrm{~h}$, annexin $V$ analyses were performed as described in the Materials and Methods. B and C: KBV20C cells were grown on 60 mm-diameter dishes and treated with $5 \mu M$ fluphenazine (FLU), lopinavir (LOP), or $10 \mu M$ verapamil (VER), alone and in combination with $50 \mathrm{ng} / \mathrm{ml}$ eribulin (ERI) (B) or $5 \mu M$ nelfinavir $(C)$, or $0.1 \%$ dimethyl sulfoxide (Con). After 1 day, all cells were examined using an inverted microscope at $\times 10$ magnification.

ritonavir can be replaced by well-known P-gp inhibitors that can inhibit P-gp with reduced toxicity in clinical settings.

In summary, we found that HIV protease inhibitors have different P-gp-inhibitory activities and different mechanisms for sensitizing vincristine-treated resistant KBV20C cells, which are P-gp overexpressing cells. Interestingly, nelfinavir sensitized vincristine-treated KBV20C cells with very low Pgp-inhibitory activity, whereas lopinavir sensitized vincristinetreated KBV20C cells with high P-gp-inhibitory activity.

Vincristine-nelfinavir co-treatment increases late apoptosis, whereas vincristine-lopinavir co-treatment increases early apoptosis in resistant KBV20C cells. Although vincristinenelfinavir and vincristine-lopinavir co-treatments had similarly high sensitizing effects on P-gp-overexpressing KBV20C cells (Figures 1E, 2A and 3A), we found that sensitization by vincristine-nelfinavir is independent of the $\mathrm{P}$-gp-inhibitory activity of nelfinavir (Figure 3B). In contrast to nelfinavir, lopinavir had higher P-gp-inhibitory activity, similarly to that of verapamil. Therefore, we assumed that vincristinenelfinavir and vincristine-lopinavir co-treatments have different sensitizing mechanisms in resistant KBV20C cells. Annexin V staining was therefore analyzed in more detail. As shown in Figure 4A, we found that vincristine-nelfinavir greatly increased late apoptosis. However, vincristineverapamil and vincristine-lopinavir co-treatment increased early apoptosis more than late apoptosis. Considering that verapamil and lopinavir have high P-gp-inhibitory activity, we conclude that the sensitizing mechanism without P-gp inhibition of the vincristine-nelfinavir combination involves late apoptosis in P-gp-overexpressing resistant KBV20C cells.

Co-treatments using vincristine with nelfinavir, lopinavir, and fluphenazine have similar sensitizing effects on resistant 
KBV20C cells. The anti-psychotic repositioned drug fluphenazine has been shown to sensitize eribulin-treated KBV20C cells (20). We tested whether the combination of nelfinavir or lopinavir with eribulin would be more effective than fluphenazine co-treatment. Microscopic observations indicated that eribulin-nelfinavir and eribulin-lopinavir cotreatments had better or similar sensitizing effects compared to those of eribulin-fluphenazine (Figure 4B), suggesting that nelfinavir, lopinavir, and fluphenazine have similar sensitization of eribulin-treated resistant cancer cells. Collectively, highly resistant KBV20C cells can be sensitized by co-treatment with repositioned drugs such as nelfinavir, lopinavir, and fluphenazine at a similar low dose.

Lastly, we confirmed that nelfinavir and lopinavir only sensitize resistant cancer cells when combined with antimitotic drug. As seen in Figure 4C, combination of nelfinavir with Pgp inhibitor verapamil or the combination of two HIV protease inhibitors (nelfinavir-lopinavir) did not increase sensitization, as these co-treatments led to similar sensitization as treatment with single drugs. This suggests that nelfinavir and lopinavir can only play a toxic role for against resistant cancer cells when combined with antimitotic drug.

We identified nelfinavir and lopinavir among five HIV protease inhibitors as having highly antimitotic-sensitizing effects on P-gp-overexpressing resistant cancer cells. Interestingly, nelfinavir appears to sensitize cells by increasing late-phase apoptosis without inhibiting P-gp.

\section{Discussion}

Drug repositioning or drug repurposing is the application of known drugs for new indications (9-11). This approach has been used for the treatment of various diseases and has advantages such as low cost and avoidance of many toxicity tests, which is a time-consuming process. The urgent need for pharmacological treatments for resistant cancer can be efficiently addressed with drug repositioning, and these drugs can be applied in clinical settings at a relatively fast pace. We have investigated and reported the application of repositioned drugs for patients with drug-resistant cancer. For example, we suggested the application of antimalarial or antipsychotic drugs in those with $\mathrm{P}$-gp-overexpressing resistant cancer $(21-23,28)$.

In our current study, we identified drugs which might be novelly repositioned for sensitizing $\mathrm{P}$-gp-overexpressing resistant cancer: Nelfinavir and lopinavir, which are HIV protease inhibitors. On testing five HIV proteases (atazanavir, nelfinavir, darunavir, lopinavir, and ritonavir), we observed that co-treatment with nelfinavir or lopinavir sensitized antimitotic drug-resistant KBV20C cells at a relatively low dose. Although co-treatment with atazanavir or ritonavir also showed sensitizing effects on vincristine-treated KBV20C cells, they required higher doses than did nelfinavir and lopinavir.
Our further studies on the mechanism involved in sensitization thus focused more on nelfinavir and lopinavir. Considering that the four HIV protease members studied had sensitizing effects on vincristine-resistant cancer cells, we conclude that HIV protease inhibitors generally have the ability to overcome resistance to antimitotic drugs. As HIV proteases are located in the cellular membrane and transport the amplified virus out of cells $(12,13)$, we assumed that HIV protease inhibitors play a role in reducing or modifying P-gp activity in the membrane of resistant cancer cells. Although the sensitizing ability of HIV protease inhibitors has been demonstrated $(18,31)$, ours is a pioneering finding of using selective HIV proteases as repositioned drugs. Considering that patients with HIV were shown to have much higher incidences of cancer (32), our finding might also contribute to the use of select HIV protease inhibitors for preventing or reducing cancer occurrence in patients with HIV.

Notably, our results were not limited to vincristine cotreatment because we confirmed that nelfinavir and lopinavir have sensitizing effects similar to those observed for eribulin in P-gp-overexpressing KBV20C cells. Eribulin was recently developed and is a promising drug for the treatment of patients in whom other anticancer drugs have failed $(29,30)$. We previously reported that P-gp-overexpressing KBV20C cells are highly eribulin-resistant $(20,25)$. Therefore, KBV20C cells are useful as a model of highly eribulin-resistant cancer. We found that nelfinavir or lopinavir can sensitize KBV20C cells to eribulin treatment. Nelfinavir and lopinavir can sensitize other antimitotic drug-resistant cancer cells and vincristineresistant KBV20C cells at similar doses. We hypothesize that nelfinavir and lopinavir can be used in combination with other cancer drugs for sensitizing resistant cancer cells.

A detailed analysis was performed to determine the molecular mechanisms underlying the sensitizing effects so that nelfinavir or lopinavir can be applied in clinical settings at a relatively fast pace, especially in patients resistant to antimitotic drugs. We demonstrated that vincristine-nelfinavir and vincristine-lopinavir co-treatment reduced cellular proliferation and increased $\mathrm{G}_{2}$ arrest in P-gp-overexpressing resistant KBV20C cells. Based on microscopic, FACS, and annexin $\mathrm{V}$ analyses, we concluded that apoptosis was increased by vincristine-nelfinavir and vincristine-lopinavir co-treatment via increased $\mathrm{G}_{2}$ arrest and reduced proliferation. Using detailed FACS analysis, we observed that nelfinavir and lopinavir, among the five tested HIV protease inhibitors, led to much higher $\mathrm{G}_{2}$ arrest in combination with vincristine, confirming that nelfinavir and lopinavir are the best choices for co-treatment with antimitotic drug in resistant cancer.

The repositioned anti-psychotic drug fluphenazine in low doses has been shown to sensitize antimitotic drug-resistant KBV20C cells (20). Therefore, we tested whether nelfinavir or lopinavir sensitized antimitotic drug-treated resistant KBV20C cells as much as fluphenazine. When we analyzed 
the sensitizing effects of nelfinavir, lopinavir, and fluphenazine, we concluded that they have similar sensitizing effects at similar doses. This suggests that the HIV protease inhibitors nelfinavir and lopinavir can be considered as novel repositioned drugs with similar effects to the well-known repositioned anti-psychotic drug fluphenazine.

As the efflux of vincristine by P-gp is the main mechanism for the resistance of $\mathrm{KBV} 20 \mathrm{C}$ cells to vincristine, we tested whether sensitization by vincristine-nelfinavir or vincristinelopinavir co-treatment resulted from the inhibitory activity of nelfinavir or lopinavir. We demonstrated that lopinavir has high P-gp-inhibitory activity, similar to that of the well-known P-gp inhibitor verapamil, suggesting that vincristine-lopinavir sensitizing results from the inhibitory activity of lopinavir preventing efflux of vincristine. Interestingly, we did not detect much P-gp-inhibitory activity by nelfinavir, suggesting that nelfinavir removes or inhibits factors that block the effects of vincristine in drug-resistant cancer cells and that vincristinenelfinavir then exerts a synergistic effect on these cotreated cells. Because we did not detect increased P-gp inhibition on treatment with nelfinavir, this may help improve combination chemotherapeutic treatments for patients with cancer which develops resistance to antimitotic drugs. P-gp inhibitors have shown toxicity to normal cells; therefore, we can consider nelfinavir as a drug without $\mathrm{P}$-gp inhibition which can be used in combination to sensitize P-gp-overexpressing resistant cancer cells. As personalized medicines become more common, our findings on nelfinavir and lopinavir might help improve prescriptions for patients with drug-resistant cancer who are allergic or sensitive to the P-gp-inhibitory effects on normal tissues.

As vincristine-nelfinavir and vincristine-lopinavir showed different mechanisms of sensitization, we tried to identify them. We found that the mechanism of sensitization without P-gp inhibition using vincristine-nelfinavir involved late apoptosis of P-gp-overexpressing resistant KBV20C cells, whereas vincristine-lopinavir largely increased early apoptosis. As sensitization by vincristine-verapamil and vincristine-lopinavir result from the high P-gp-inhibitory activity of verapamil and lopinavir, we conclude that the sensitizing mechanism without P-gp inhibition using vincristine-nelfinavir involves late apoptosis of P-gp-overexpressing resistant KBV20C cells. We assume that the quick induction of late apoptosis by vincristinenelfinavir improves cytotoxicity towards resistant cancer cells without allowing them time to recover from the drug's effects. A study has shown that doxorubicin-resistant cancer cells can be sensitized by co-treatment with nelfinavir, which plays a role in P-gp inhibition to prevent efflux of doxorubicin (19). However, our results showed that the mechanism of action of vincristine-nelfinavir sensitization does not involve P-gpinhibition by nelfinavir, as compared to the role of lopinavir (with high P-gp-inhibitory activity) in sensitization by vincristine-lopinavir. Further investigation of nelfinavir may be needed for resistant cancer types depending on the organ of origin, anticancer drugs in use, or drug-injection times.

Taken together, our results highlight the novel selective sensitization by HIV protease inhibitors. Our results show that drug-resistant KBV20C cells that overexpress P-gp can be sensitized to antimitotic drug treatment (eribulin or vincristine) with repositioned drugs (nelfinavir or lopinavir) at low doses. Notably, nelfinavir sensitized drug-resistant cancer cells without P-gp-inhibitory activity. As their toxicity profile is already known, these drugs are readily available for clinical use. Our results contribute to the improvement of the efficacy of various chemotherapeutic agents used in combination for the treatment of patients with cancer who develop resistance to chemotherapeutic drugs via P-gp overexpression.

\section{Conflicts of Interest}

The Authors declare no conflicts of interest regarding this study.

\section{Authors' Contributions}

JYK: Collected the data, contributed data or analysis tools, wrote the article. YJP: collected the data, wrote the article. B-ML: contributed data or analysis tools. SY: conceived and designed the analysis, collected the data, contributed data or analysis tools, wrote the article.

\section{Acknowledgements}

This research was supported by National Research Foundation of Korea (NRF) funded by the Ministry of Education (NRF2017R1D1A1B03029158).

\section{References}

1 Jordan MA and Wilson L: Microtubules as a target for anticancer drugs. Nat Rev Cancer 4: 253-265, 2004. PMID: 15057285. DOI: $10.1038 / \mathrm{nrc} 1317$

2 Kim JH, Yoo HI, Kang HS, Ro J and Yoon S: Salinomycin sensitizes antimitotic drugs-treated cancer cells by increasing apoptosis via the prevention of G2 arrest. Biochem Biophys Res Commun 418: 98-103, 2012. PMID: 22244892. DOI: 10.1016/j.bbrc.2011.12.141

3 McGrogan BT, Gilmartin B, Carney DN and McCann A: Taxanes, microtubules and chemoresistant breast cancer. Biochim Biophys Acta 1785: 96-132, 2008. PMID: 18068131. DOI: $10.1016 /$ j.bbcan.2007.10.004

4 Szakacs G, Paterson JK, Ludwig JA, Booth-Genthe C and Gottesman MM: Targeting multidrug resistance in cancer. Nat Rev Drug Discov 5: 219-234, 2006. PMID: 16518375. DOI: $10.1038 / \operatorname{nrd} 1984$

5 Chen Z, Shi T, Zhang L, Zhu P, Deng M, Huang C, Hu T, Jiang $\mathrm{L}$ and Li J: Mammalian drug efflux transporters of the ATP binding cassette $(\mathrm{ABC})$ family in multidrug resistance: A review of the past decade. Cancer Lett 370: 153-164, 2016. PMID: 26499806. DOI: 10.1016/j.canlet.2015.10.010

6 Chufan EE, Kapoor K and Ambudkar SV: Drug-protein hydrogen bonds govern the inhibition of the ATP hydrolysis of the multidrug 
transporter P-glycoprotein. Biochem Pharmacol 101: 40-53, 2016. PMID: 26686578. DOI: 10.1016/ j.bcp.2015.12.007

7 Shukla S, Wu CP and Ambudkar SV: Development of inhibitors of ATP-binding cassette drug transporters: Present status and challenges. Expert Opin Drug Metab Toxicol 4: 205-223, 2008. PMID: 18248313. DOI: 10.1517/17425255.4.2.205

8 Yang K, Wu J and Li X: Recent advances in the research of Pglycoprotein inhibitors. Biosci Trends 2: 137-146, 2008. PMID: 20103919.

9 Clark KB: New therapeutic bearings for repositioned drugs. Curr Top Med Chem 13: 2281-2282, 2013. PMID: 24059466.

10 Yoon S: A single treatment of Selenate, a repositioning drug, specifically sensitizes P-gp-overexpressing resistant cancer cells. Cancer Cell Microenv 2(4), 2015. DOI: http://dx.doi.org/ 10.14800/ccm.957

11 Pantziarka P and Cairns L: Recycling existing drugs for cancer therapy: delivering low cost cancer care. E Cancer Med Sci 8: ed40, 2014. PMID: 25075221. DOI: 10.3332/ecancer.2014.ed40

12 Bierman WF, Scheffer GL, Schoonderwoerd A, Jansen G, van Agtmael MA, Danner SA and Scheper RJ: Protease inhibitors atazanavir, lopinavir and ritonavir are potent blockers, but poor substrates, of $\mathrm{ABC}$ transporters in a broad panel of $\mathrm{ABC}$ transporter-overexpressing cell lines. J Antimicrob Chemother 65 : 1672-1680, 2010. PMID: 20551216. DOI: $10.1093 /$ jac/dkq209

13 Cerveny L, Ptackova Z, Durisova M and Staud F: Interactions of protease inhibitors atazanavir and ritonavir with $\mathrm{ABCB} 1$, $\mathrm{ABCG} 2$, and $\mathrm{ABCC} 2$ transporters: Effect on transplacental disposition in rats. Reprod Toxicol 79: 57-65, 2018. PMID: 29859254. DOI: 10.1016/j.reprotox.2018.05.008

14 Coburger C, Lage H, Molnar J, Langner A and Hilgeroth A: Multidrug resistance reversal properties and cytotoxic evaluation of representatives of a novel class of HIV-1 protease inhibitors. J Pharm Pharmacol 62: 1704-1710, 2010. PMID: 21054396. DOI: $10.1111 / \mathrm{j} .2042-7158.2010 .01144 . x$

15 Katoh M, Nakajima M, Yamazaki H and Yokoi T: Inhibitory effects of CYP3A4 substrates and their metabolites on Pglycoprotein-mediated transport. Eur J Pharm Sci 12: 505-513, 2001. PMID: 11231118.

16 Zastre JA, Chan GN, Ronaldson PT, Ramaswamy M, Couraud PO, Romero IA, Weksler B, Bendayan M and Bendayan R: Upregulation of $\mathrm{P}$-glycoprotein by HIV protease inhibitors in a human brain microvessel endothelial cell line. J Neurosci Res 87: 1023-1036, 2009. PMID: 18855943. DOI: 10.1002/jnr.21898

17 Zhou SF: Drugs behave as substrates, inhibitors and inducers of human cytochrome P450 3A4. Curr Drug Metab 9: 310-322, 2008. PMID: 18473749.

18 Lucia MB, Anu R, Handley M, Gillet JP, Wu CP, De Donatis GM, Cauda R and Gottesman MM: Exposure to HIV-protease inhibitors selects for increased expression of P-glycoprotein (ABCB1) in Kaposi's sarcoma cells. Br J Cancer 105: 513-522, 2011. PMID: 21829205. DOI: 10.1038/bjc.2011.275

19 Chakravarty G, Mathur A, Mallade P, Gerlach S, Willis J, Datta A, Srivastav S, Abdel-Mageed AB and Mondal D: Nelfinavir targets multiple drug resistance mechanisms to increase the efficacy of doxorubicin in MCF-7/Dox breast cancer cells. Biochimie 124: 53 64, 2016. PMID: 26844637. DOI: 10.1016/j.biochi.2016.01.014

20 Cheon JH, Lee BM, Kim HS and Yoon S: Highly halavenresistant KBV20C cancer cells can be sensitized by co-treatment with fluphenazine. Anticancer Res 36: 5867-5874, 2016. PMID: 27793910. DOI: 10.21873/anticanres.11172
21 Kim JY, Park Y, Lee BM, Kim HS and Yoon S: P-gp inhibition by the anti-psychotic drug pimozide increases apoptosis, as well as expression of $\mathrm{pRb}$ and $\mathrm{pH} 2 \mathrm{AX}$ in highly drug-resistant KBV20C Cells. Anticancer Res 38: 5685-5692, 2018. PMID: 30275188. DOI: 10.21873 /anticanres. 12905

22 Kim JY, Son JY, Lee BM, Kim HS and Yoon S: Aging-related repositioned drugs, donepezil and sildenafil citrate, increase apoptosis of anti-mitotic drug-resistant KBV20C cells through different molecular mechanisms. Anticancer Res 38: 5149-5157, 2018. PMID: 30194162. DOI: 10.21873/anticanres.12837

23 Kim JY, Tae IH, Lee BM, Kim HS and Yoon S: Low doses of the anti-psychotic drug aripiprazole have strong P-gp-inhibitory activity and sensitize anti-mitotic drug-resistant cancer cells. Anticancer Res 38: 5101-5108, 2018. PMID: 30194155. DOI: 10.21873/anticanres.12830

24 Cheon JH, Kim JY, Lee BM, Kim HS and Yoon S: P-gp Inhibition by XL019, a JAK2 inhibitor, increases apoptosis of vincristine-treated resistant KBV20C cells with increased p21 and pH2AX expression. Anticancer Res 37: 6761-6769, 2017. PMID: 29187454. DOI: 10.21873/anticanres.12136

25 Cheon JH, Kim KS, Yadav DK, Kim M, Kim HS and Yoon S: The JAK2 inhibitors CEP-33779 and NVP-BSK805 have high P-gp-inhibitory activity and sensitize drug-resistant cancer cells to vincristine. Biochem Biophys Res Commun 490: 1176-1182, 2017. PMID: 28669723. DOI: 10.1016/j.bbrc.2017.06.178

26 Park Y, Son JY, Lee BM, Kim HS and Yoon S: Highly eribulinresistant KBV20C oral cancer cells can be sensitized by cotreatment with the third-generation P-glycoprotein inhibitor, elacridar, at a low dose. Anticancer Res 37: 4139-4146, 2017. PMID: 28739698. DOI: 10.21873/anticanres.11801

27 Choi AR, Kim JH, Cheon JH, Kim HS and Yoon S: Attenuation of colchicine toxicity in drug-resistant cancer cells by cotreatment with anti-malarial Drugs. Anticancer Res 36: 58595866, 2016. PMID: 27793909. DOI: 10.21873/anticanres.11171

28 Choi AR, Kim JH, Woo YH, Kim HS and Yoon S: Anti-malarial drugs primaquine and chloroquine have different sensitization effects with anti-mitotic drugs in resistant cancer cells. Anticancer Res 36: 1641-1648, 2016. PMID: 27069141.

29 Dybdal-Hargreaves NF, Risinger AL and Mooberry SL: Eribulin mesylate: Mechanism of action of a unique microtubuletargeting agent. Clin Cancer Res 21: 2445-2452, 2015. PMID: 25838395. DOI: 10.1158/1078-0432.CCR-14-3252

30 Inoue K, Saito T, Okubo K, Kimizuka K, Yamada H, Sakurai T, Ishizuna K, Hata S, Kai T and Kurosumi M: Phase II clinical study of eribulin monotherapy in Japanese patients with metastatic breast cancer who had well-defined taxane resistance. Breast Cancer Res Treat 157: 295-305, 2016. PMID: 27125669. DOI: $10.1007 / \mathrm{s} 10549-016-3808-\mathrm{x}$

31 Chow WA, Jiang C and Guan M: Anti-HIV drugs for cancer therapeutics: back to the future? Lancet Oncol 10: 61-71, 2009. PMID: 19111246. DOI: 10.1016/S1470-2045(08)70334-6

32 Gobert A, Veyri M, Poizot-Martin I, Lavolé A, Solas C, Paliche R, Katlama C, Costagliola D and Spano JP: HIV and cancer: What's new in 2017? Bull Cancer 105: 256-262, 2018. PMID: 29548534. DOI: $10.1016 /$ j.bulcan.2018.02.002

Received April 5, 2019

Revised May 7, 2019

Accepted May 8, 2019 